\title{
Acute Encephalopathy as a Presenting Symptom of Nontyphoidal Salmonellosis in Children
}

\author{
Jithin C Shaji ${ }^{1}$, Remesh Kumar ${ }^{2}$
}

\begin{abstract}
In this case report, we describe a child who had fever and encephalopathy at the time of presentation. Nontyphoidal salmonellosis causing encephalopathy in children is scarcely described in the literature, and in those, concurrent abdominal symptoms were also well manifested. In our report, the abdominal findings were rather absent, and most clinical symptoms pointed toward a CNS infection. Active CNS infection was ruled out through CSF examination and the culprit for encephalopathy was found out through blood culture. Through this case report, we want to make the practicing clinicians aware of the possibility of nontyphoidal salmonellosis to present as encephalopathy alone without any obvious abdominal symptoms to suspect the disease.
\end{abstract}

Keywords: Blood culture, Children, Encephalopathy, Gastroenteritis, Nontyphoidal salmonellosis.

Pediatric Infectious Disease (2021): 10.5005/jp-journals-10081-1291

\section{BACKGROUND}

Nontyphoidal salmonellosis refers to illnesses caused by all other serotypes of Salmonella except typhi, paratyphi A, paratyphi B (tartrate negative), and paratyphi C. The most common clinical presentation of this illness is gastroenteritis manifesting usually as acute diarrhea, abdominal pain, fever, and vomiting. Encephalopathy has been believed to be an unusual manifestation of nontyphoidal Salmonella (NTS) infection. However, it should be considered as an important cause of encephalopathy as sometimes diffuse and rapidly progressive brain dysfunction and circulatory shock may ensue after NTS infection. Here, we describe a case where the abdominal manifestations of this disease were not evident but manifested only as an encephalopathy. Our patient might have contracted salmonellosis from raw milk.

\section{Case Description}

A previously healthy 1-year-old girl child, with no past history of febrile convulsions and no family history of febrile seizures/epilepsy, was admitted in pediatric intensive care unit with fever, altered sensorium, and refractory generalized tonic seizures. Her fever started 1 day earlier. She was not started on any antibiotics. No loose stools were present. There was no history of any accidental ingestion. On admission here, she had altered sensorium, the Glasgow coma scale (GCS) of only $5 / 15$, and was seizing. No signs of dehydration were present. Neurological examination showed no focal deficits. CRP was positive (59), and blood electrolytes including blood sugar were within normal limits. Arterial blood gas and renal function were also normal. Transaminases were mildly elevated. The CSF analysis showed no meningitis. Her seizures controlled with inj. lorazepam, inj. fosphenytoin, and inj. levetiracetam. She was started on inj. ceftriaxone and inj. acyclovir. Encephalopathy continued for 1 day and her sensorium improved over the next few days. EEG and MRI brain were normal. The CSF culture was sterile. Blood C\&S yielded nontyphoidal salmonella susceptible to ceftriaxone. Retrospective history taken from parents showed that \begin{tabular}{l}
\hline 1,2Department of Pediatrics, Apollo Adlux Hospital, Kochi, Kerala, India \\
Corresponding Author: Jithin C Shaji, Department of Pediatrics, \\
Apollo Adlux Hospital, Kochi, Kerala, India, Phone: +917592837693 , \\
e-mail: jithin15@yahoo.com \\
How to cite this article: Shaji JC, Kumar R. Acute Encephalopathy as \\
a Presenting Symptom of Nontyphoidal Salmonellosis in Children. \\
Pediatr Inf Dis 2021;3(2):81-82. \\
Source of support: Nil \\
Conflict of interest: None
\end{tabular}

they used to give raw milk from a local cow farm. Inj. acyclovir was stopped later as the viral panel came as negative.

\section{Discussion}

The above case report suggests the possibility of bacteremia due to nontyphoidal salmonellosis as a significant cause for encephalopathy in a small child. Only a few literatures in the past have reported NTS encephalopathy ${ }^{1}$ in children and hence the importance of this report. Gastroenteritis symptoms were apparent in almost all previous literatures unlike our case report. Secondary factors such as severe dehydration, sepsis, meningitis, electrolyte or metabolic disturbances, acute renal failure, and multiple organ failure were considered in the differential diagnosis at the onset of encephalopathic features. NTS encephalopathy is rarely accompanied by abnormalities in laboratory data. Investigations done for this child have ruled out above possibilities as other potential causes for encephalopathy.

It also suggests the likely role of nontyphoidal salmonella to cause an encephalopathy that is very much similar to other known diarrheal pathogens like Shigella and E. coli. Shigellosis ${ }^{2}$ and other Shiga toxin-producing bacteria such as E. coli $0157^{3}$ are known to produce encephalopathy, though the mechanism is unclear.

The exact etiology by which salmonella causes encephalopathy is still unknown. However, various etiologies from endotoxemia ${ }^{4}$ to cytotoxic factors like L-asparaginase ${ }^{1,5}$ have been postulated. Other 
unknown factors apart from cytotoxic substances could also be involved in the mechanism of encephalopathy.

\section{CONCLUSION}

Culturing organism continues to be the mainstay of clinical diagnostic testing for NTS. Preventive measures should include food and water precautions, such as avoiding foods and drinks at high risk for contamination and maintaining hand hygiene, especially after contact with animals or their environment.

\section{Clinical Significance}

This case report should make clinicians aware that nontyphoidal salmonellosis can present with encephalopathy alone without any noticeable abdominal manifestations, in which situation culturing organism through blood would help to identify the offending pathogen.

\section{References}

1. Arii J, Tanabe $Y$, Miyake $M$, et al. Clinical and pathological characteristics of nontyphoidal salmonella encephalopathy. Neurology 2002;58(11):1641-1645. DOI: 10.1212/WNL.58.11.1641.

2. Perles Z, BarZiv J, Granot E. Brain edema: an underdiagnosed complication of shigella infection. Pediatr Infect Dis J 1995;14(12):11141115. DOI: $10.1097 / 00006454-199512000-00022$.

3. Kleanthous H, Smith HR, Scotland SM, et al. Haemolytic uraemic syndromes in the British Isles, 1985-8: association with verocytotoxin producing Escherichia coli. part 2: microbiological aspects. Arch Dis Child 1990;65(7):722-727. DOI: 10.1136/adc.65.7.722.

4. Martin K, Sharland M, Davies E. Encephalopathy associated with Salmonella enteritidis infection. Scand J Infect Dis 1994;26(4):486-488. DOI: 10.3109/00365549409008624.

5. Iwamaru Y, Miyake M, Arii J, et al. An inhibitory factor for cell-free protein synthesis from Salmonella enteritidis exhibits cytopathic activity against Chinese hamster ovary cells. Microb Pathog 2001;31(6):283-293. DOI: 10.1006/mpat.2001.0471. 